DePauw University

Scholarly and Creative Work from DePauw University

6-2021

\title{
Mediators of the relationship between self-control and pathological technology use: Negative affect and cognitive failures, but not self-efficacy
}

Robert West

DePauw University, robertwest@depauw.edu

Diana Jiang

DePauw University

Follow this and additional works at: https://scholarship.depauw.edu/psyc_facpubs

Part of the Neuroscience and Neurobiology Commons

\section{Recommended Citation}

West, R., \& Jiang, D. (2021). Mediators of the relationship between self-control and pathological technology use: Negative affect and cognitive failures, but not self-efficacy. In F. D. Davis et al. (Eds.), Information Systems and Neuroscience, 220-228, Lecture Notes in Information Systems and Organisation. https://dx.doi.org/10.1007/978-3-030-88900-5_25

This Conference Proceeding is brought to you for free and open access by the Psychology and Neuroscience at Scholarly and Creative Work from DePauw University. It has been accepted for inclusion in Psychology and Neuroscience Faculty Publications by an authorized administrator of Scholarly and Creative Work from DePauw University. 


\title{
Proceedings NeuroIS Retreat 2021
}

\author{
Virtual Conference | June 1-3 \\ www.NeuroIS.org
}

Fred D. Davis, René Riedl, Jan vom Brocke, Pierre-Majorique Léger, Adriane B. Randolph, Gernot Müller-Putz (Eds.)

The final proceedings will be published by Springer. 


\title{
Preface
}

The proceedings contain papers presented at the 13th annual NeuroIS Retreat held June 1-3 2021. NeuroIS is a field in Information Systems (IS) that uses neuroscience and neurophysiological tools and knowledge to better understand the development, adoption, and impact of information and communication technologies (www.neurois.org).

The NeuroIS Retreat is a leading academic conference for presenting research and development projects at the nexus of IS and neurobiology. This annual conference promotes the development of the NeuroIS field with activities primarily delivered by and for academics, though works often have a professional orientation.

In 2009 the inaugural NeuroIS Retreat was held in Gmunden, Austria. Since then, the NeuroIS community has grown steadily, with subsequent annual Retreats in Gmunden from 2010-2017. Beginning in 2018, the conference is taking place in Vienna, Autria. Due to the Corona crisis, the organizers decided to host a virtual NeuroIS Retreat in 2021.

The NeuroIS Retreat provides a platform for scholars to discuss their studies and exchange ideas. A major goal is to provide feedback for scholars to advance their research papers toward high-quality journal publications. The organizing committee welcomes not only completed research, but also work in progress. The NeuroIS Retreat is known for its informal and constructive workshop atmosphere. Many NeuroIS presentations have evolved into publications in highly regarded academic journals.

This year is the seventh time that we publish the proceedings in the form of an edited volume. A total of 27 research papers were accepted and published in this volume, and we observe diversity in topics, theories, methods, and tools of the contributions in this book. The 2021 keynote presentation entitled "Decision Neuroscience: How it started and where we are today" is given by Antoine Bechara, professor of neuroscience and psychology at the University of Southern California (USC) in Los Angeles, USA. Moreover, Moritz Grosse-Wentrup, professor and head of the Research Group Neuroinformatics at the University of Vienna, Austria, gives a hot topic talk entitled "How (not) to interpret Multivariate Decoding Models in Neuroimaging".

Altogether, we are happy to see the ongoing progress in the NeuroIS field. Also, we can report that the NeuroIS Society, established in 2018 as a non-profit organization, has been developing well. We foresee a prosperous development of NeuroIS.

June 2021

\author{
Fred D. Davis \\ René Riedl \\ Jan vom Brocke \\ Pierre-Majorique Léger \\ Adriane B. Randolph \\ Gernot Müller-Putz
}




\section{Sponsors}

We thank the sponsors of the NeuroIS Retreat 2021:

Main Sponsors
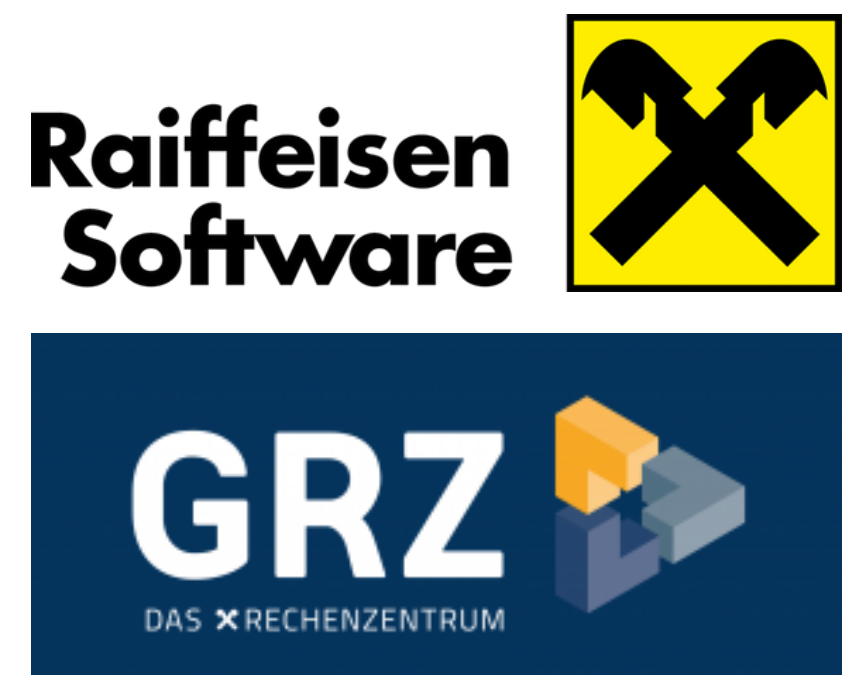

Further Sponsors
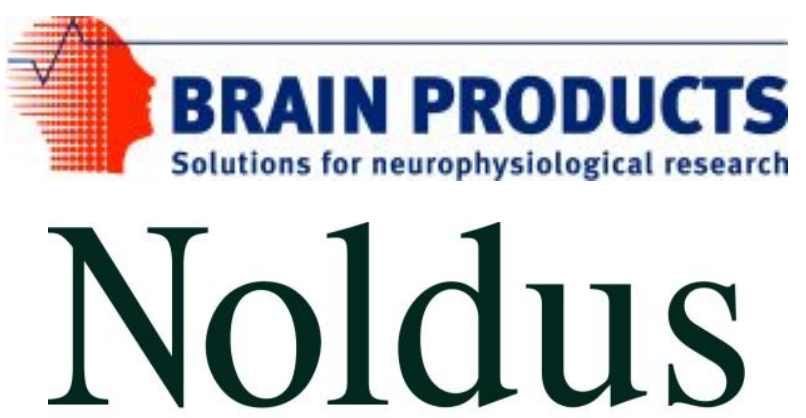

Information Technology 


\title{
Antoine Bechara - Keynote
}

\author{
Decision Neuroscience: How It Started and Where We Are Today
}

Decision neuroscience is an emerging area of research whose goal is to integrate research in neuroscience and behavioral decision-making. Neuroeconomics is a more specialized field of study that seeks to bridge neuroscience research on human choice with economic theory, whereas neuromarketing addresses the neuroscience behind consumers' choices, including product branding, preference, and purchase decisions. More recent research seeks to include the field of information science by examining the impact of social media and other technology use on the human brain. All these areas capitalize on knowledge from the fields of neuroscience, behavioral economics, finances, marketing, and information science to explore the neural "road map" for the physiological processes intervening between knowledge and behavior, and the potential interruptions that lead to a disconnection between what one knows and what one decides to do. Thus, decision neuroscience is the domain that captures the interests of scientists who are attempting to understand the neural basis of judgment and decisionmaking in health as well as social behavior.

\section{Moritz Grosse-Wentrup - Hot Topic Talk}

\section{How (not) to Interpret Multivariate Decoding Models in Neuroimaging}

Multivariate decoding models are replacing traditional univariate statistical tests in the analysis of neuroimaging data. Their interpretation, however, is far from trivial. In this presentation, I outline various pitfalls and discuss under which conditions they can provide insights into the (causal) question of how neuronal activity gives rise to cognition and behavior.

\section{Panel Discussion}

\section{Success Factors in Publishing NeuroIS Research in Top IS Journals: Experiences of MIS Quarterly Editors and Reviewers}

Moderator: Fred D. Davis

Panelists: Ofir Turel, Tony Vance, Adriane B. Randolph, Eric Walden 


\section{Table of Contents}

Where NeuroIS Helps to Understand Human Processing of Text: A Taxonomy for Research Questions Based on Textual Data.

Towards a Psychophysiological Investigation of Perceived Trustworthiness and Risk

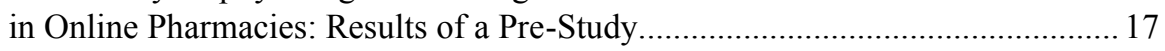

Exploring the Influence of Personality Traits on Affective Customer Experiences in Retailing: Combination of Heart Rate Variability (HRV) and Self-Report Measures

Motor Dysfunction Simulation in Able-Bodied Participants for Usability Evaluation of Assistive Technology: A Research Proposal ....................................................... 39

Exploring the Potential of NeuroIS in the Wild: Opportunities and Challenges of Home Environments 48

Exploring the Recognition of Facial Activities Through Around-the-Ear Electrode Arrays (cEEGrids).

Leveraging NeuroIS Tools to Understand Consumer Interactions with Social Media Content 66

Optimizing Scatterplot-Matrices for Decision-Support: An Experimental EyeTracking Study Assessing Situational Cognitive Load ...................................... 73

“Overloading” Cognitive (Work)Load: What are We Really Measuring? .................. 86

On Electrode Layout in EEG Studies: A Limitation of Consumer-Grade EEG Instruments.

Predicting In-Field Flow Experiences Over Two Weeks from ECG Data: A Case Study

An Inward Focus of Attention During Information Security Decision Making: Electrophysiological Evidence

EyeTC: Attentive Terms and Conditions of Internet-based Services with WebcamBased Eye Tracking

Detecting Flow Experiences in the Field Using Video-Based Head and Face Activity Recognition: A Pilot Study ..... 134

Understanding the Potential of Augmented Reality in Manufacturing Environments 142

On How Mind Wandering Facilitates Creative Incubation While Using Information Technology: A Research Agenda for Robust Triangulation 155

Consumers Prefer Abstract Design in Digital Signage: An Application of Fuzzy-Trace Theory in NeuroIS 164 
Topographic Analysis of Cognitive Load in Tacit Coordination Games Based on Electrophysiological Measurements .............................................................. 180

Active Learning Techniques for Preparing NeuroIS Researchers ........................... 191

Examining the Impact of Social Video Game Tournaments on Gamers' Mental WellBeing 197

Continuing Doctoral Student Training for NeuroIS and EEG During a Pandemic: A Distance Hands-On Learning Syllabus

Design Mode, Color, and Button Shape: A Pilot Study on the Neural Effects of Website Perception

Does Media Richness Influence the User Experience of Chatbots: A Pilot Study ... 225

Development of a New Dynamic Personalised Emotional Baselining Protocol for Human-Computer Interaction

Mediators of the Relationship Between Self-Control and Pathological Technology Use: Negative Affect and Cognitive Failures, But Not Self-Efficacy....

High Fidelity Vibrokinetic Stimulation Augments Emotional Reactivity and Interhemispheric Coherence During Passive Multimedia Interaction.

Explainable Artificial intelligence (XAI): How the Visualization of AI Predictions Affects User Cognitive Load and Confidence 


\title{
Where NeuroIS Helps to Understand Human Processing of Text: A Taxonomy for Research Questions Based on Textual Data
}

\author{
Florian Popp, Bernhard Lutz, and Dirk Neumann \\ University of Freiburg, Freiburg, Germany florianmaximilianpopp@gmail.com \\ \{bernhard.lutz,dirk.neumann\}@is.uni-freiburg.de
}

\begin{abstract}
Several research questions from information systems (IS) are based on textual data, such as product reviews and fake news. In this paper, we investigate in which areas NeuroIS is best suited to better understand human processing of text and subsequent human behavior or decision making. To evaluate this question, we propose a taxonomy to distinguish these research questions depending on how users' corresponding response is formed. We first review all publications about textual data in the IS basket journals from 2010-2020. Then, we distinguish text-based research questions along two dimensions, namely, if a user's response is influenced by subjectivity and if additional information is required to make an objective assessment. We find that NeuroIS research on textual data is still in its infancy. Existing NeuroIS studies focus on texts, where users' responses are subject to a higher need for additional data, which is not part of the text.
\end{abstract}

Keywords: Textual data - Taxonomy · Information processing - Decision-making · NeuroIS

\section{Introduction}

Social media and other forms of computer-mediated communication provide users with large amounts of textual data [1]. Text provides an unstructured and highdimensional source of information as English texts can easily comprise vocabularies with tens of thousands of words [2]. So far, IS research has analyzed how users respond to texts of many types, such as fake news, online reviews, financial reports, spam emails, and computer-mediated communication. While the factors of a helpful product review are well understood (e.g., [3-5]), little is known of why users fall for deception in the form of fake news. Deciding if a news article is real or fake may be subjective and depends on prior beliefs and attitudes [6-8]. Making an objective veracity assessment of a news article may also require additional information from external sources, which is not part of the actual text [9]. Whether or not users invest cognitive effort into a critical assessment also depends on their mindset. Users in a utilitarian mindset are used to spending cognitive effort in reading the text as part of their daily (working) routines, whereas users in a hedonic mindset (e.g., when being active on social media) are less likely to carefully reflect on their actions [6]. As a 
consequence, their behavior may be driven by unconscious processes, which can be studied by employing tools and theories from NeuroIS [10-13].

One possible reason for the limited understanding of specific IS phenomena based on textual data is that users' responses may exhibit higher degrees of subjectivity or that making an objective assessment requires additional information, which is not part of the text. In this study, we propose a taxonomy that distinguishes research questions based on textual data according to whether a user's response is driven by a low or high degree of subjectivity and the need for additional information in order to make an objective judgement. We specifically build our taxonomy for the research questions instead of the type of text themselves. The underlying reasoning is that the same type of text can be subject to different research questions. For instance, a news article can be categorized as positive or negative, or as real or fake. However, categorizing the article as positive or negative is less affected by political opinions and unconscious processes than categorizing an article as real or fake. The proposed taxonomy was developed based on all articles about text-related research questions from the IS basket journals published between 2010 and 2020. We categorized each of these studies along the dimensions "degree of subjectivity" and "need for additional information". In addition, we distinguish all studies according to (1) whether they collected userspecific data to account for individual beliefs and attitudes, and (2) studies that can be attributed to the field of NeuroIS. Taken together, we address the following research question:

"For which research questions based on textual data should researchers employ methods from NeuroIS?"

\section{Literature Review}

For our literature review about research on textual data, we consider all articles published in the IS basket journals between 2010 and 2020. We first read the title of all issues to determine whether an article is about textual data or not. Subsequently, we read the abstract of the remaining studies to validate our initial assessment. Thereby, we encountered 47 studies about text. We excluded nine of these studies [14-22] as they did not specifically analyze human behavior as a direct response to reading the text. Regarding the publication dates, we find that the number of studies about textual data and human responses has increased over the last few years. While 10 out of 38 papers were published in 2010-2015, the majority of 28 were published in the years 2016-2020. Finally, we scan the remaining 38 papers in regard to their collected data. We distinguish between (1) studies without user-specific data (e.g., political attitude, disposition to trust), (2) studies with user-specific data, and (3) studies with NeuroIS measurements.

Table 1 shows the result of our literature review. We identified four studies from NeuroIS, from which three $([6,23,24])$ are published in MIS Quarterly (MISQ) and one ([25]) in the European Journal of Information Systems (EJIS). Moravec et al. [6] analyzed the influence of IS design modifications on users' cognition when processing fake news. In their experiment, subjects were shown several online news 
articles with and without warning messages, while their cognitive activity was measured with electroencephalography (EEG). The authors found that adding warning messages increases cognitive activity, but this has no impact on the final veracity assessment. Bera et al. [23] used eye tracking to explain cognitive processing of users in reading conceptual modeling scripts to perform problem solving tasks. Their results suggest that high task performance can be explained and detected by attention-based eye tracking metrics. Meservy et al. [24] studied how individuals evaluate and filter posts in online forums, while seeking the solution to a problem. For this purpose, the authors employed functional magnetic resonance imaging (fMRI) to measure the neural correlates that are involved in evaluating both solution content and contextual cues. Their results indicate that both content and contextual cues impact users' final filtering decisions. Finally, Brinton Anderson et al. [25] used text mining and eye tracking to study how users perceive security messages and found that habituation to security warnings significantly reduces the effect of such warnings.

Table 1. Studies about textual data in IS basket journals from 2010 to 2020 .

\begin{tabular}{lllll}
\hline Journal & No user-specific data & User-specific data & NeuroIS & Total \\
\hline JMIS & {$[26-38]$} & {$[8]$} & & 14 \\
MISQ & {$[39-44]$} & {$[7]$} & {$[6,23,24]$} & 10 \\
JAIS & {$[45-49]$} & {$[50]$} & 6 \\
ISR & {$[5,51-53]$} & & 4 \\
JIT & {$[54,55]$} & & 2 \\
JSIS & {$[56]$} & & 1 \\
EJIS & & & $125]$ & 1 \\
ISJ & & 3 & 4 & 0 \\
\hline Total & 31 & & 48
\end{tabular}

We identified three studies which collected user-specific data, but without employing tools and theories from NeuroIS. For instance, Hong et al. [50] used a combination of surveys and text mining in order to explain and predict deviations in restaurant reviews based on cultural differences. Their findings suggest that users from a collectivist culture tend to agree with prior ratings. Kim and Dennis [8] collected users' political attitude and studied whether fake news can be mitigated by adding 
user and expert ratings to the presentation of a news article. Their results suggest that expert ratings have a stronger impact in steering users to a correct veracity assessment. However, this only holds when the rating indicates low reliability, whereas ratings of high reliability have no effect. In another study on fake news, Kim et al. [7] studied the effects of alternating the presentation format. Their results suggest that presenting the source before the headline (instead of showing the headline before the source) makes users less likely to perceive fake news as real. In addition, the authors find that source reputation ratings have a stronger effect on the perceived believability. However, this only holds when the rating is low (i.e., indicating fake content).

All other studies did not collect user-specific data as part of their research design. Instead, they rely on statistical methods from text mining to empirically analyze how users respond to textual data. These studies covered consumer behavior [30, 33, 39, 40, 48, 49, 51, 53, 55, 56], financial decision-making [29, 31, 32, 35, 36, 43, 46, 47, $54]$, perception of online reviews [5, 26-28, 42, 45], deception detection [37, 38], and others $[41,52]$.

\section{Taxonomy for Research Questions Based on Text}

We now present our taxonomy to distinguish research questions based on textual data depending on whether users' response is influenced by subjectivity and if there is a need for additional information in order to make a correct assessment. Hereby, "degree of subjectivity" refers to the questions: How subjectively can the probands answer the (research) question? Would people with different prior experience or belief answer differently? "Need for additional information" on the other hand, refers to the questions: Would a proband's answer become better or more correct if they had additional information or additional knowledge? Is all relevant information that is needed for the proband to complete the task or answer the (research) question given in the text? Or is additional information, knowledge, or background required? We propose this taxonomy in order to address our research question. Based on the following cluster analysis, we identify areas in which NeuroIs seems best suited in order to understand human processing of text and their subsequent behavior or decision making? Figure 1 presents the proposed taxonomy along these two dimensions. All studies including user specific data from surveys are highlighted with a square, while all NeuroIS studies are highlighted with a circle. For the purpose of this study, we distinguish all studies from our literature based on our own reading. Overall, the NeuroIS studies were located in the upper part of Figure 1, which contains those research questions, where users have a higher need for additional information. The upper right corner of Figure 1 contains all studies analyzing research questions, where users' responses are driven by a high degree of subjectivity with a high need for additional information to make an objective assessment. Prominent examples are studies that analyze why users fall for fake news $[6,7,7]$. The decision if a news article is real of fake depends on users' individual political attitudes, while making an objective assessment generally requires users to research additional information. 


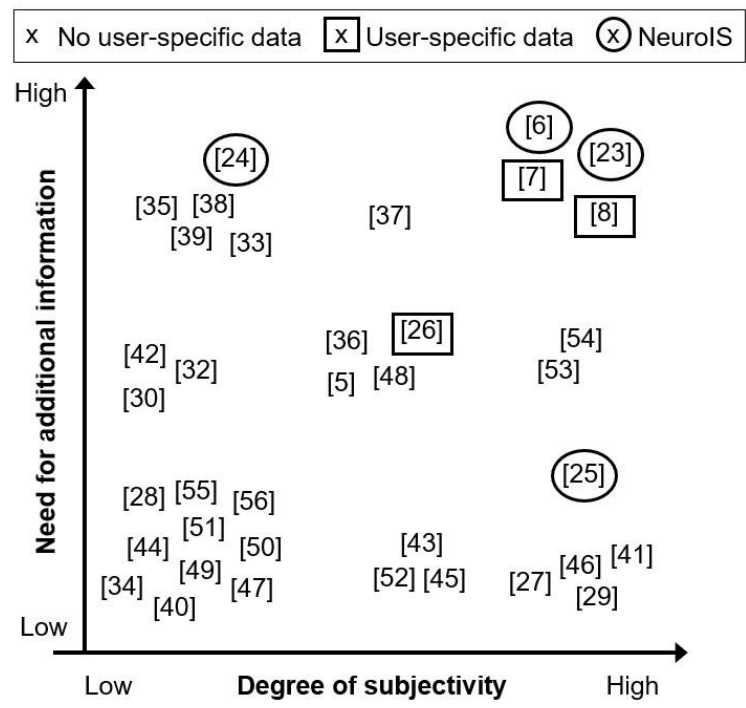

Fig.1. Taxonomy to distinguish research questions based on textual data depending on the characteristics of users' responses.

The studies in the bottom right area of Figure 1 consider research questions, where users' responses involve high degree of subjectivity without a need for additional information. One example is to explain why social media posts receive more likes (e.g., [40]). The decision of whether to like a social media post or not is subjective as it depends on personal tastes, while all relevant information based on which users decide to like a post is generally included in the text. The upper left corner of Figure 1 contains all studies analyzing research questions, where users' responses are driven by low degree of subjectivity, but with a high need for additional information. We classified all studies about fraud or deception detection as such [32, 34, 37, 38, 47]. Deciding if a message originates from a deceiver is generally not driven by personal attitudes. However, the decision is often made based on incomplete information. Ultimately, the studies in the bottom left area of Figure 1 consider research questions, where users' responses are not influenced by subjectivity without a need for additional information. For instance, this applies to the question of how daily deals impact a restaurant's online reputation [51]. The review text generally stands for itself, while the factors of a positive or negative review (length, number of arguments, high readability) are usually not influenced by subjectivity.

\section{Conclusion and Future Research}

Understanding how humans respond to textual data provides novel challenges for IS research and practice. We proposed a taxonomy to distinguish different forms of texts after reviewing recent studies from the IS literature according to their research design. In this taxonomy, we distinguish research questions based on textual data with respect 
to the characteristics of users' responses. Specifically, we consider two dimensions, namely how much a user's response is driven by subjectivity and the need for additional information in order to make an objective assessment. We find that NeuroIS is used for research questions, where users arrive at conclusions based on incomplete information. All corresponding studies [6, 23-25] focused on users' cognitive processing. It appears that this is the area, where NeuroIS is best equipped to help understand human processing of text and help explain human behavior and decision making. However, the vast majority of existing studies in the IS literature considered texts where users' responses can be explained without user-specific data.

IS research on textual data should hence select their study design depending on the characteristics of users' responses. If users' conclusions exhibit only little subjectivity and the text contains all relevant information, there is no need for experiments and employing measurements from neuroscience. Conversely, if users' responses are driven by a higher degree of subjectivity and if additional information is required for an objective assessment, users may rely on unconscious and/or heuristic processes. For example, affective processes may not be fully reflected through self-reports, which requires IS researchers to employ neurophysiological measurements such as ECG or startle reflex modulation [13].

We plan to extend this study as follows. First, we need to evaluate our taxonomy by gathering feedback from external domain experts. Presenting our work at the NeuroIS retreat would be one important step in this direction. After this, we can perform a sequence of evaluation-improvement iterations until we reach an acceptable state. Second, we aim to extend our literature review to all studies in the existing IS literature to provide a full overview of IS studies on textual data. Third, we are planning to elaborate more on the specific tools from NeuroIS, in particular, how specific measurements can be applied to study users' responses to different types of text. 


\section{References}

1. Debortoli, S., Mu“1ler, O., Junglas, I., Vom Brocke, J.: Text mining for information systems researchers: An annotated topic modeling tutorial. Communications of the Association for Information Systems 39, 110-135 (2016)

2. Gentzkow, M., Kelly, B., Taddy, M.: Text as data. Journal of Economic Literature 57(3), 535-574 (2019)

3. Mudambi, S.M., Schuff, D.: What makes a helpful online review? A study of customer reviews on amazon.com. MIS Quarterly 34(1), 185-200 (2010)

4. Korfiatis, N., Garc'ia-Bariocanal, E., S'anchez-Alonso, S.: Evaluating content quality and helpfulness of online product reviews: The interplay of review helpfulness vs. review content. Electronic Commerce Research and Applications 11(3), 205-217 (2012)

5. Yin, D., Mitra, S., Zhang, H.: When do consumers value positive vs. negative reviews? An empirical investigation of confirmation bias in online word of mouth. Information Systems Research 27(1), 131-144 (2016)

6. Moravec, P., Kim, A., Dennis, A., Minas, R.: Fake news on social media: People believe what they want to believe when it makes no sense at all. MIS Quarterly 43(4), 1343-1360 (2019)

7. Kim, A., Moravec, P.L., Dennis, A.R.: Combating fake news on social media with source ratings: The effects of user and expert reputation ratings. Journal of Management Information Systems 36(3), 931-968 (2019)

8. Kim, A., Dennis, A.: Says who? The effects of presentation format and source rating on fake news in social media. MIS Quarterly 43(3), 1025-1039 (2019)

9. Graves, L.: Boundaries not drawn: Mapping the institutional roots of the global fact-checking movement. Journalism Studies 19(5), 613-631 (2016)

10. Dimoka, A., Pavlou, P.A., Davis, F.D.: NeuroIS: The potential of cognitive neuroscience for information systems research. Information Systems Research 22(4), 687-702 (2011)

11. Dimoka, A., Davis, F.D., Gupta, A., Pavlou, P.A., Banker, R.D., Dennis, A.R., Ischebeck, A., Mu“ller-Putz, G., Benbasat, I., Gefen, D., et al.: On the use of neurophysiological tools in IS research: Developing a research agenda for NeuroIS. MIS Quarterly 36(3), 679-702 (2012)

12. Riedl, R., Fischer, T., L'eger, P.M., Davis, F.D.: A decade of NeuroIS research: Progress, challenges, and future directions. DATA BASE for Advanced Information Systems pp. 1-51 (2019)

13. vom Brocke, J., Hevner, A., L'eger, P.M., Walla, P., Riedl, R.: Advancing a neurois research agenda with four areas of societal contributions. European Journal of Information Systems 29(1), 9-24 (2020)

14. Safi, R., Yu, Y.: Online product review as an indicator of users' degree of innovativeness and product adoption time: A longitudinal analysis of text reviews. European Journal of Information Systems 26(4), 414-431 (2017) 
15. Abbasi, A., Li, J., Adjeroh, D., Abate, M., Zheng, W.: Don't mention it? analyzing user-generated content signals for early adverse event warnings. Information Systems Research 30(3), 1007-1028 (2019)

16. Mejia, J., Mankad, S., Gopal, A.: A for effort? Using the crowd to identify moral hazard in new york city restaurant hygiene inspections. Information Systems Research 30(4), 1363-1386 (2019)

17. Liu, Y., Pant, G., Sheng, O.R.: Predicting labor market competition: Leveraging interfirm network and employee skills. Information Systems Research 31(4), 1443-1466 (2020)

18. Shi, D., Guan, J., Zurada, J., Manikas, A.: A data-mining approach to identification of risk factors in safety management systems. Journal of Management Information Systems 34(4), 1054-1081 (2017)

19. Vlas, R.E., Robinson, W.N.: Two rule-based natural language strategies for requirements discovery and classification in open source software development projects. Journal of Management Information Systems 28(4), 11-38 (2012)

20. Hu, Y., Xu, A., Hong, Y., Gal, D., Sinha, V., Akkiraju, R.: Generating business intelligence through social media analytics: Measuring brand personality with consumer-, employee-, and firm-generated content. Journal of Management Information Systems 36(3), 893-930 (2019)

21. Zhou, S., Qiao, Z., Du, Q., Wang, G.A., Fan, W., Yan, X.: Measuring customer agility from online reviews using big data text analytics. Journal of Management Information Systems 35(2), 510-539 (2018)

22. Wang, Z., Jiang, C., Zhao, H., Ding, Y.: Mining semantic soft factors for credit risk evaluation in peer-to-peer lending. Journal of Management Information Systems 37(1), 282-308 (2020)

23. Bera, P., Soffer, P., Parsons, J.: Using eye tracking to expose cognitive processes in understanding conceptual models. MIS Quarterly 43(4), 1105-1126 (2019)

24. Meservy, T.O., Fadel, K.J., Kirwan, C.B., Meservy, R.D.: An fMRI exploration of information processing in electronic networks of practice. MIS Quarterly 43(3), 851-872 (2019)

25. Brinton Anderson, B., Vance, A., Kirwan, C.B., Eargle, D., Jenkins, J.L.: How users perceive and respond to security messages: A NeuroIS research agenda and empirical study. European Journal of Information Systems 25(4), 364-390 (2016)

26. Huang, L., Tan, C.H., Ke, W., Wei, K.K.: Comprehension and assessment of product reviews: A review-product congruity proposition. Journal of Management Information Systems 30(3), 311-343 (2013)

27. Ma, X., Khansa, L., Deng, Y., Kim, S.S.: Impact of prior reviews on the subsequent review process in reputation systems. Journal of Management Information Systems 30(3), 279-310 (2013)

28. Jensen, M.L., Averbeck, J.M., Zhang, Z., Wright, K.B.: Credibility of anonymous online product reviews: A language expectancy perspective. Journal of Management Information Systems 30(1), 293-324 (2013) 
29. Luo, X., Zhang, J.: How do consumer buzz and traffic in social media marketing predict the value of the firm? Journal of Management Information Systems 30(2), 213-238 (2013)

30. Ghiassi, M., Zimbra, D., Lee, S.: Targeted twitter sentiment analysis for brands using supervised feature engineering and the dynamic architecture for artificial neural networks. Journal of Management Information Systems 33(4), 1034-1058 (2016)

31. Lash, M.T., Zhao, K.: Early predictions of movie success: The who, what, and when of profitability. Journal of Management Information Systems 33(3), 874903 (2016)

32. Siering, M., Koch, J.A., Deokar, A.V.: Detecting fraudulent behavior on crowdfunding platforms: The role of linguistic and content-based cues in static and dynamic contexts. Journal of Management Information Systems 33(2), 421-455 (2016)

33. Huang, J., Boh, W.F., Goh, K.H.: A temporal study of the effects of online opinions: Information sources matter. Journal of Management Information Systems 34(4), 1169-1202 (2017)

34. Ho, S.M., Hancock, J.T., Booth, C., Liu, X.: Computer-mediated deception: Strategies revealed by language-action cues in spontaneous communication. Journal of Management Information Systems 33(2), 393-420 (2016)

35. Mai, F., Shan, Z., Bai, Q., Wang, X., Chiang, R.H.: How does social media impact bitcoin value? a test of the silent majority hypothesis. Journal of Management Information systems 35(1), 19-52 (2018)

36. Dong, W., Liao, S., Zhang, Z.: Leveraging financial social media data for corporate fraud detection. Journal of Management Information Systems 35(2), 461-487 (2018)

37. Ludwig, S., Van Laer, T., De Ruyter, K., Friedman, M.: Untangling a web of lies: Exploring automated detection of deception in computer-mediated communication. Journal of Management Information Systems 33(2), 511- 541 (2016)

38. Liang, N., Biros, D.P., Luse, A.: An empirical validation of malicious insider characteristics. Journal of Management Information Systems 33(2), 361-392 (2016)

39. Huang, N., Hong, Y., Burtch, G.: Social network integration and user content generation: Evidence from natural experiments. MIS Quarterly pp. 17-001 (2016)

40. Shin, D., He, S., Lee, G.M., Whinston, A.B., Cetintas, S., Lee, K.C.: Enhancing social media analysis with visual data analytics: A deep learning approach. MIS Quarterly 44(4), 1459-1492 (2020)

41. Ludwig, S., De Ruyter, K., Mahr, D., Wetzels, M., Bru"ggen, E., De Ruyck, T.: Take their word for it. MIS Quarterly 38(4), 1201-1218 (2014)

42. Susan, M.M., David, S.: What makes a helpful online review? A study of customer reviews on amazon. com. MIS Quarterly 34(1), 185-200 (2010) 
43. Deng, S., Huang, Z.J., Sinha, A.P., Zhao, H.: The interaction between microblog sentiment and stock return: An empirical examination. MIS Quarterly 42(3), 895-918 (2018)

44. Yin, D., Bond, S.D., Zhang, H.: Anxious or angry? Effects of discrete emotions on the perceived helpfulness of online reviews. MIS Quarterly 38(2), 539-560 (2014)

45. Kuan, K.K., Hui, K.L.: What makes a review voted? An empirical investigation of review voting in online review systems. Journal of the Association for Information Systems 16(1), 48-71 (2015)

46. Ho, S.Y., Choi, K.W.S., Yang, F.F.: Harnessing aspect-based sentiment analysis: How are tweets associated with forecast accuracy? Journal of the Association for Information Systems 20(8), 2 (2019)

47. Lausen, J., Clapham, B., Siering, M., Gomber, P.: Who is the next "wolf of wall street"? detection of financial intermediary misconduct. Journal of the Association for Information Systems 21(5), 7 (2020)

48. Chou, C.H., Sinha, A.P., Zhao, H.: A hybrid attribute selection approach for text classification. Journal of the Association for Information Systems 11(9), 1 (2010)

49. Chen, Y., Deng, S., Kwak, D.H., Elnoshokaty, A., Wu, J.: A multi-appeal model of persuasion for online petition success: A linguistic cue-based approach. Chen, Y., Deng, S., Kwak, DH, Elnoshokaty, A., \& Wu, J (2019)

50. Hong, Y., Huang, N., Burtch, G., Li, C.: Culture, conformity and emotional suppression in online reviews. Journal of the Association for Information Systems pp. 16-020 (2016)

51. Bai, X., Marsden, J.R., Ross Jr, W.T., Wang, G.: A note on the impact of daily deals on local retailers' online reputation: Mediation effects of the consumer experience. Information Systems Research 31(4), 1132-1143 (2020)

52. Pan, Y., Huang, P., Gopal, A.: Storm clouds on the horizon? New entry threats and $R \& D$ investments in the US IT industry. Information Systems Research 30(2), 540-562 (2019)

53. Adamopoulos, P., Ghose, A., Todri, V.: The impact of user personality traits on word of mouth: Text-mining social media platforms. Information Systems Research 29(3), 612-640 (2018)

54. Li, T., van Dalen, J., van Rees, P.J.: More than just noise? examining the information content of stock microblogs on financial markets. Journal of Information Technology 33(1), 50-69 (2018)

55. Garcia-Crespo, A., Colomo-Palacios, R., Gomez-Berbis, J.M., Ruiz-Mezcua, B.: Semo: A framework for customer social networks analysis based on semantics. Journal of Information Technology 25(2), 178-188 (2010)

56. Benthaus, J., Risius, M., Beck, R.: Social media management strategies for organizational impression management and their effect on public perception. The Journal of Strategic Information Systems 25(2), 127-139 (2016) 


\title{
Mediators of the Relationship Between Self-Control and Pathological Technology Use: Negative Affect and Cognitive Failures, But Not Self-Efficacy
}

\author{
Robert West, and Diana Jiang \\ Department of Psychology and Neuroscience, DePauw University, Greencastle, \\ USA \\ robertwest@depauw.edu,diana_Jiang_@outlook.com
}

\begin{abstract}
The widespread adoption of technologies such as smartphones, the Internet, and social media has been associated with the emergence of pathological technology use (e.g., Internet addiction). Prevalence rates of pathological technology use vary widely across age groups, cultures, and medium, although it is not uncommon for rates of mild to moderate pathological use to exceed $20 \%-30 \%$. These relatively high prevalence rates have motivated researchers to identify the predictors of pathological use. The current study focuses on the relationship between self-control and pathological technology use, and demonstrates that negative affect and cognitive failures, but not self-efficacy, partially mediate the association between self-control and pathological technology use. These findings reveal some of the pathways by which poor self-control could lead to elevated levels of pathological technology use.
\end{abstract}

Keywords: self-control, negative affect, pathological technology use

\section{Introduction}

The widespread adoption of smartphones and the technologies that they provide access to (e.g., the Internet and social media) over the last two decades has revolutionized the way we interact with friends and family, conduct business, pursue an education, and spend our leisure time [1]. In contrast to the many benefits these technologies have brought to our lives, there are also significant personal and social costs associated with the abusive (e.g., cyberbullying) and pathological (e.g., Internet addiction) use of technology [2-3]. Estimates of the prevalence of pathological technology use vary widely across medium, although it is not uncommon for rates to fall between $20 \%-30 \%$ or higher depending upon the sample and form of technology [46]. These high prevalence rates have motivated extensive work seeking to identify the causes and consequences of pathological technology use [3,6,7]. This research reveals that pathological technology use is consistently associated with diminished selfcontrol [8] and poor mental health outcomes including increased depression, anxiety, 
stress, and suicidal ideation [9]. The current study sought to extend upon the extant literature by examining whether the relationships between aspects of self-control and pathological technology use (i.e., smartphone, Internet, social media) are partially or fully mediated through effects on negative affect, cognition, and self-efficacy.

Studies have consistently demonstrated that poor self-control is associated with an increase in pathological technology use [7] and addiction more generally [10]. As an example, Kim et al. [11] found that individual differences in self-control and impulsivity were unique predictors of smartphone pathology. Consistent with this finding, individuals with attention deficit hyperactivity disorder (ADHD) experience higher levels of pathological smartphone use than typically developing individuals [12]. Poor self-control may also account for the association between pathological technology use including video games [13], social media [14], and the Internet [15] and poor decision making in the Iowa Gambling Task (IGT) reported in a number of studies. These findings are consistent with work using electrophysiological and neuroimaging methods demonstrating that pathological technology use is associated with attenuated neural activity related to reward processing [16] and to reduced brain volume in a number of structures within the reward network [17]. The disruption of neural activity and alternations in brain volume and connectivity within the fronto-striatal reward/control network are commonly implicated in disorders of self-control, emotion dysregulation, and addiction [18].

Self-control is a complex construct that represents aspects of impulsivity, risktaking, and emotion regulation $[19,20]$. Poor self-control is associated with increases in negative affect (i.e., depression, anxiety, stress), poor cognitive control, and reduced self-efficacy [19,21-22], and each of these are themselves associated with pathological technology use. Therefore, an important question represents the degree to which the influence of self-control on pathological technology use may be mediated through its influence on negative affect, cognitive control, and self-efficacy. To address this question, the current study examined three hypotheses related to possible mediators of the relationship between self-control and pathological technology use:

H1: Poor self-control is associated with increases in negative affect, cognitive failures, pathological technology use, and decreases in self-efficacy.

H2: Negative affect, cognitive failures, and self-efficacy are associated with pathological technology use.

H3: The relationship between self-control and pathological technology use is mediated through negative affect, cognitive failures, and self-efficacy.

To examine these hypotheses, individuals completed measures of pathological technology use (i.e., smartphone, Internet, social media), a multidimensional measure of self-control, a measure of negative affect tapping depression, anxiety and stress, and a measure of cognitive failures. We did not include a measure of pathological Internet gaming as in some previous research an effect of gender has been observed in this domain $[12,23]$, wherein pathology is much more common in males than in females, an interaction we hoped to avoid in the current dataset. The multidimensional measure of self-control allowed us to distinguish between the influence of aspects of self-control that were differentially related to impulsivity and emotion regulation, and risk-taking on pathological technology use. 


\section{Method}

\section{Participants}

The sample included 210 individuals from the Prolific registry. To participate in the study, individuals had to be 18 years of age or older and have an IP address registered in the United States. The average age of the sample was 31 years ( $\mathrm{SD}=11$ years), with $43 \%$ females, $49 \%$ males, $4 \%$ gender non-conforming, $2 \%$ trangender, and $1 \%$ other. The sample was predominantly white (63\%), followed by Asian (13\%), Black or African American (11\%), Hispanic or Latinx (10\%), Indigenous (1\%), and other (2\%); $19 \%$ had not completed any college, $31 \%$ had completed $1-2$ years of college, $8 \%$ had completed 3 years of college, $28 \%$ had completed 4 years of college, and $14 \%$ had completed some graduate training. Individuals were compensated \$5 US for participation in the study through their Prolific account.

\section{Materials and Procedure}

Cronbach's $\boldsymbol{\alpha}$ was used to estimate the reliability (internal consistency) of the scales for the data obtained for the study, these values are reported in the text. The self-control scale measures six aspects of self- control (Impulsivity $\boldsymbol{\alpha}=.82$, Risktaking $\boldsymbol{\alpha}=.85$, Self concern $\boldsymbol{\alpha}=.78$, a preference for Simple tasks $\boldsymbol{\alpha}=.87$, a preference for Physical activity over mental activity $\boldsymbol{\alpha}=.78$, Tempe (emotion regulation) $\boldsymbol{\alpha}=.80)[20]$; for the Self-control scale higher scores represent lower self-control. Pathological technology use was measures with the Internet Addiction Test-Short Version $(\boldsymbol{\alpha}=.86)[24]$, Smartphone Addiction Scale-Short Version (SAS-SV, $\boldsymbol{\alpha}=.87$ ) [25], and Bergen Social Media Addiction Scale (BSMAS, $\boldsymbol{\alpha}=.86$ )[26]. Negative affect was measured with the Depression, Anxiety and Stress Scale-21 (DASS-21Depression $\boldsymbol{\alpha}=.93$, Anxiety $\boldsymbol{\alpha}=.86$, Stress $\boldsymbol{\alpha}=.89$ )[27]. The Cognitive Failures Questionnaire (CFQ, $\boldsymbol{\alpha}=.94)[28]$ was used to assess cognitive control. Self-efficacy was measured with the General Self-Efficacy Scale (GSF, $\boldsymbol{\alpha}=.91$ ) [29] and Short Grit Scale (perseverance subscale $\boldsymbol{\alpha}=.76$ ) [30]. Individuals also completed demographic measures including age, gender, education, and race and ethnicity, and other scales not relevant to the current project. There were three attention check questions distributed across the study materials, these included "Which of the words is presented in upper case letters? - cat, RAT, bat, sat; "Which of the words is a color?" - red, grass, sky, water; "Which word is an animal?" - water, pan, cup, fox.

Individuals completed the study through a link posted on the Prolific website. The study materials were presented using Google Forms. At the beginning of the study, individuals read a consent form and checked a box indicating their willingness participant in the study and then continued with the survey materials. Completion of the study materials required 15 to 30 minutes across participants. 


\section{Data Cleaning and Preparation}

All participants answered the three attention check questions correctly and were therefore retaining in the sample. Some participants were missing responses for an item from an individual scale or subscale. These missing values were imputed with the participants mean for the scale or subscale, thereby allowing us to estimate reliability in the entire sample without impacting individual scores for the measures or the pattern of correlation across the measures. For the analyses, scale level measures were formed by calculating the average response across items for each of the scales or subscales (Self-control scale). Additionally, composite measures were formed by averaging scores for the three pathology measures (smartphone, internet, social media pathology) and the three negative affect measures (depression, anxiety, stress) given the higher degree of correlation among the relevant measures.

\section{Results}

Scores for the three pathology measures were highly correlated, as was the case for the measures of negative affect (Table 1). Self-efficacy and perseverance for the Grit scale were also highly correlated $(r=.58, \mathrm{p}<.001)$; therefore, a composite variable was formed representing the average Z-score for the two measures as they are on different scales. The six subscales of the self-control scale revealed a mixed pattern of correlations (Table 2). A principal component analysis [31] was performed for the Self-control scale in an effort to reduce the number of variables included in the analyses, while capturing the underlying structure within the six subscales. Consistent with previous research [32], the PCA revealed two components with Eigenvalues greater than 1.0 that accounted for $61 \%$ of the variance, and were moderately correlated $(r=.24)$. The first component ( $\mathrm{SC} 1)$ represented impulsivity (component loading $=.76)$, self-concern (component loading $=.64)$, simple task (component loading $=$ .84 ), and tempe (component loading $=.72$ ), and the second component (SC2) represented risk-taking (component loading $=.68$ ) and physical tasks (component loading $=.87$ ). Two composite variables were formed that represented the average of impulsivity, self-concern, simple task, and tempe, or risk- taking and physical tasks.

Supporting Hypotheses 1 and 2, the correlations between pathology and the predictors were medium to large, except for the SC2 composite where the correlation was small (Table 3); all correlations were significant. Pathology increased with greater negative affect and cognitive failures, and less self-control, and decreased with greater self-efficacy. The correlations between the SC1 composite and the mediators were also medium to large, and significant. The correlations between the SC2 composite and the mediators were small, and the correlation with negative affect was not significant. 
Table 1. Pearson correlations between measures of pathology (left side) and negative affect (right side)

\begin{tabular}{|c|c|c|c|c|c|c|c|}
\hline Pathology & 1 & 2 & 3 & $\begin{array}{l}\text { Negative Af- } \\
\text { fect }\end{array}$ & 4 & 5 & 6 \\
\hline 1. Internet & & & & $\begin{array}{l}\text { 4. Depres- } \\
\text { sion }\end{array}$ & & & \\
\hline $\begin{array}{l}2 . \\
\text { Smartphone }\end{array}$ & 69 & & & 5. Anxiety & 71 & & \\
\hline $\begin{array}{c}\text { 3. } \\
\text { Media }\end{array}$ & 63 & .71 & & 6. Stress & 71. & .82 & \\
\hline
\end{tabular}

Note: All correlations are significant at $\mathrm{p}<.001$.

Table 2. Pearson correlations among the subscales of the self-control scale.

\begin{tabular}{|c|c|c|c|c|c|c|}
\hline & 1 & 2 & 3 & 4 & 5 & 6 \\
\hline 1. Imp. & -- & & & & & \\
\hline 2. Risk & $\begin{array}{l}.40^{*} \\
*\end{array}$ & -- & & & & \\
\hline 3. Self & $\begin{array}{l}.29 * \\
*\end{array}$ & $.22 *$ & -- & & & \\
\hline ple ${ }^{4 . \quad \text { Sim- }}$ & $\begin{array}{l}.51^{*} \\
*\end{array}$ & .05 & $.35^{* *}$ & -- & & \\
\hline $\begin{array}{l}\text { 5. Phys- } \\
\text { ical }\end{array}$ & .006 & $.29 * *$ & $.15 \#$ & $-.16 \#$ & -- & \\
\hline 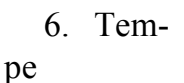 & $\begin{array}{l}.40^{*} \\
*\end{array}$ & $.26^{* *}$ & $.50 * *$ & $.35 * *$ & .05 & -- \\
\hline
\end{tabular}

Note: $\# \mathrm{p}<.05, * \mathrm{p}<.01, * * \mathrm{p}<.001$ 
Table 3. Pearson correlations between pathology and the predictor variables.

\begin{tabular}{|c|c|c|c|c|c|c|}
\hline \multicolumn{7}{|l|}{-} \\
\hline 1.Pathology & 1 & 2 & 3 & 4 & 5 & 6 \\
\hline 2. SC 1 & $\underset{* *}{.50}$ & & & & & \\
\hline 3. SC 2 & $\begin{array}{l}.17 \\
\#\end{array}$ & $.21^{*}$ & & & & \\
\hline $\begin{array}{l}\text { 4. Negative Af- } \\
\text { fect }\end{array}$ & $\underset{* *}{.52}$ & $\begin{array}{l}.48 * \\
*\end{array}$ & .11 & & & \\
\hline 5. CFQ & $\begin{array}{l}.46 \\
* *\end{array}$ & $\begin{array}{l}.46^{*} \\
*\end{array}$ & $.19 *$ & $.57 * *$ & & \\
\hline 6. Self-efficacy & $.31^{* *}$ & $.45^{-}$ & $.17 *$ & $-.35 * *$ & $.31^{-}$ & \\
\hline
\end{tabular}

Note: $\# \mathrm{p}<.05, * \mathrm{p}<.01, * * \mathrm{p}<.001$

We tested a set of four mediation models to determine whether the relationship between self-control and pathology was fully or partially mediated by negative affect, cognitive failures, or self-efficacy. The models were fit using the Mediation Model in JASP (0.14, JASP Team 2020) based upon the Lavaan SEM package with R [33]. Maximum likelihood estimation was used. For the direct and indirect effects, we report the z-test for the parameter estimates along with the $95 \%$ confidence interval based upon 1000 Bootstrap samples with the Bias-corrected percentile [34]. An initial set of models including both components of self-control revealed that the total effect of SC2 was not significant $(p=.28)$. Therefore, this variable was dropped from the analyses to simplify the models. The individual mediator models provided partial support for Hypothesis 2, revealing that both negative affect and cognitive failures were partial mediators of the relationship between self-control and pathology (Table 4). In contrast, although self-efficacy was correlated with both self-control and pathology, the indirect effect was not significant in the self-efficacy model. When negative affect and cognitive failures were included in a combined model, there were significant unique indirect effects for both mediators from self-control to pathology (Table 4, Full model). 
Table 4. Standardized path coefficients, z-test, and bootstrap $95 \%$ confidence interval for the individual and combined mediator models. The direct effect from SC1 to pathology and the indirect effects through the mediators.

\begin{tabular}{|c|c|c|c|c|c|}
\hline & $\begin{array}{l}\text { Direct ef- } \\
\text { fect }\end{array}$ & z & $\begin{array}{l}\text { Indirect ef- } \\
\text { fect }\end{array}$ & $\mathrm{z}$ & R-squared \\
\hline Affect & $\begin{array}{l}.34[.22- \\
.46]\end{array}$ & $\begin{array}{l}5.18 \\
* *\end{array}$ & $\begin{array}{l}.18[.11- \\
.27]\end{array}$ & $\begin{array}{l}4.58 \\
* *\end{array}$ & .35 \\
\hline Cognition & $\begin{array}{l}.36[.25- \\
.52]\end{array}$ & $\begin{array}{l}5.73 \\
* *\end{array}$ & $\begin{array}{l}.14[.07- \\
.23]\end{array}$ & $\begin{array}{l}3.84 \\
* *\end{array}$ & .32 \\
\hline $\begin{array}{l}\text { Self effi- } \\
\text { cacy }\end{array}$ & $\begin{array}{l}.47[.32- \\
.62]\end{array}$ & $\begin{array}{l}6.80 \\
* *\end{array}$ & $\begin{array}{l}.05[-.02- \\
.14]\end{array}$ & 1.58 & .26 \\
\hline Full model & $\begin{array}{l}.30[.17- \\
.44]\end{array}$ & $\begin{array}{l}4.46 \\
* *\end{array}$ & & & .37 \\
\hline Affect & & & $\begin{array}{l}.14[.06- \\
.23]\end{array}$ & $\begin{array}{l}3.52 \\
* *\end{array}$ & \\
\hline Cognition & & & $\begin{array}{l}.08[.006- \\
.17]\end{array}$ & $\begin{array}{l}2.24 \\
\#\end{array}$ & \\
\hline
\end{tabular}

Note: $\# \mathrm{p}=.025, * \mathrm{p}<.01, * * \mathrm{p}<.001$. The $95 \%$ confidence intervals for the Bootstrap for direct and indirect effects are reported in the [].

\section{Discussion}

Here we replicated the finding that lower self-control is associated with increased levels of pathological technology use in an adult, community-based sample, with roughly equal numbers of males and females [7]. Our measure of self-control represented two distinct, but correlated, factors. One that was primarily related to impulsivity and emotion regulation (tempe) and one that was primarily related to risk taking. The impulsivity/emotion regulation component was more strongly related to pathology technology use and the three predictors (i.e., negative affect, cognitive failures, and self-efficacy) than the risk-taking component. Additionally, the impulsivity/emotion regulation component was a unique predictor of pathological technology use, while risk- taking was not. These findings provide some refinement of our 
understanding of the nature of the relationship between pathological technology use and self-control by demonstrating a specific association with impulsivity/emotion regulation. This finding is interesting within the broader literature indicating that pathological technology use may in some instances serve a compensatory role in the context of anxiety and negative affect [35].

We also observed that pathological technology use was associated with elevated negative affect and cognitive failures, and lower self-efficacy. These findings are consistent with previous research [5,11-12] and provide the foundation for testing the hypotheses related to mediation of the association between self-control and pathological technology use. The mediation analyses revealed that negative affect and cognitive failures were unique partial mediators of the association between self-control and pathological technology use. In contrast, self-efficacy did not mediate the relationship between self-control and smartphone pathology, although it was significantly correlated with both variables. This finding may indicate that high self-efficacy could provide a buffer against pathological technology that operates outside of the influence of self-control. These findings move beyond the simple observation of associations between self-control and pathological technology use by revealing potential pathways by which this association is manifest. For instance, the mediated path from selfcontrol to negative affect to pathology, may reveal how a reduction in emotion regulation could lead to increased depression, anxiety and stress, that in turn leads individuals to utilize the Internet in an attempt to boost their mood. Likewise, poor impulse control may contribute to lower cognitive control (i.e., increased cognitive failures) making it difficult to resist the temptation to frequently check social media $[19,22]$.

The current findings may be of interest to Information Systems researchers and professionals alike in demonstrating that there are multiple routes to understanding, and possibly disrupting, the link between self-control and pathological technology use. As an example, based upon our findings, intervention programs designed to reduce pathological technology use may be most successful when focused on both the affective and cognitive aspects of self-control. This suggestion is consistent with some literature examining factors that led to both the emergence of pathological technology use within individuals and also one's ability to overcome pathological use [35]. For instance, in this study individuals reported that pathological use was related to reducing negative affect or creating positive affect consistent with the link for selfcontrol to negative affect to pathological use. Also, others reported that intrinsic and extrinsic motivation that could be related to both self-control and self-efficacy were important in overcoming pathological technology use. Our findings are also interesting within the context of programs designed to disrupt pathological technology use that have a focus on providing resources for monitoring and limiting use [36], possibly serving as a surrogate for endogenous self-control. Together, the current findings and extant literature may demonstrate the important interplay between research designed to both characterize and then reduce pathological technology use.

There are some limitations of the current research worth considering. First, the three measures of pathological technology use were highly correlated, making it difficult to examine unique predictors of different forms of pathology as has been done in some previous research [23]. This might be possible with a larger sample that would 
be appropriate for estimating what are likely to be small unique effects and would also allow us to examine potential gender differences across the three forms of technology considered in the study. Second, the measures of negative affect were also highly correlated, making it difficult to consider possible unique influences of depression, anxiety, and stress. Again, a larger sample could be useful in addressing this limitation. Third, given the nature of the dataset it is impossible to fully understand the direction or nature of the causal effects reflected in the correlations observed between self-control, negative affect, cognitive failures, and pathological technology use, although we may be able to conclude that the effects of self-control and selfefficacy arise from different sources. The direction of the causal effects could be examined with either longitudinal studies over short or long periods of time, or through targeted intervention studies.

In conclusion, the current study provides a number of insights into the relationship between self-control and pathological technology use. First, we demonstrated that pathological technology use is more strongly related to aspects of impulsivity and emotion regulation rather than risk-taking within the context of self-control. Second, we observed that negative affect and cognitive failures, but not self-efficacy, were partial mediators of the association between self-control and pathological technology use. Together our findings lead to the suggestion that there are multiple pathways by which variation in self-control is associated with pathological technology use. 


\section{References}

1. Pew Research Center Infographic https://www.pewresearch.org/internet/fact-sheet/mobile/

2. Bartlett, C.., Gentile, D.: Attacking Others Online: The Formation of Cyberbullying in Late Adolescence. Psychol. Pop. Media Cult. 1, 123-135 (2012)

3. Gentile, D. A., Coyne, S. M., Bricolo, F. (2013). Pathological Technology Addictions: What is Scientifically Known and What Remains to be Learned. In: Dill, K.E. (ed.) Oxford library of psychology. The Oxford handbook of media psychology, pp. 382-402. Oxford University Press, UK (2013)

4. Gentile, D. A., Bailey, K., Bavelier, D., Brockmyer, J. F., Cash, H., Coyne, S. M., et al.: Internet Gaming Disorder in Children and Adolescents. Pediatrics, 140(Supplement 2), S81-S85 (2017)

5. Gutiérrez, J., Rodríguez de Fonseca, F., Gabriel Rubio.: Cell Phone Addiction: A Review. Front. Psychiatry. 7, 175 (2016)

6. Cash, H., Rae, C. D., Steel, A. H., Winkler, A.: Internet Addiction: A Brief Summary of Research and Practice. Curr. Psychiatry Rev. 8, 292-298 (2012).

7. Ning, W., Davis, F., Taraban, R.: Smartphone Addiction and Cognitive Performance of College Students. Twenty-fourth Americas Conference on Information Systems (2018)

8. Kim, H-J., Min, J-Y., Min, K-B., Lee, T-J., Yoo, S.: Relationship Among Family Environment, Self-control, Friendship Quality, and Adolescents' Smartphone Addiction in South Korea: Findings from Nationwide Data. PloS One 13(2): e0199896 (2018)

9. Kim, H-J., Min, J-Y., Kim, H-J., Min, K-B.: Association Between Psychological and Self-assessed Health Status and Smartphone Overuse Among Korean College Students. J. Ment. Health. doi: 10.1080/09638237.2017.1370641 (2017)

10. Sayette, M.: Self-regulatory Failure and Addiction. In: Baumeister, R., Vohs, K. (eds.) Handbook of Self-Regulation: Research, Theory, and Applications, pp. 447466. The Guilford Press, New York (2004)

11. Kim, Y., Jeong, J-E., Cho, H., Jung, D-J., Kwak, M., Rho, M. J., Yu, H., Kim, DJ., Choi, I.: Personality Factors Predicting Smartphone Addiction Predisposition: Behavioral Inhibition and Activation Systems, Impulsivity, and Self-control. PloS One, 11(8):e0159788 (2016)

12. Kim, J-H.: Psychological Issues and Problematic Use of Smartphone: ADHD's Moderating Role in the Association Among Loneliness, Need for Social Assurance, Need for Immediate Connection, and Problematic Use of Smartphone. Comput. Hum. Behav. 80, 390-398 (2018)

13. Bailey, K., West, R., \& Kuffel, J. What Would My Avatar Do? Gaming, Pathology, and Risky Decision Making. Front. Psychol. 4, Article 609, 1-10 (2013)

14. Meshi, D., Elizarova, A., Bender, A., Verdejo-Garcia, A.: Excessive Social Media Users Demonstrate Impaired Decision Making in the Iowa Gambling Task. J. Behav. Addict. 8, 169-173 (2019) 
15. Sun, D-L., Chen, Z-J., Ma, N., Zhang, X-C., Fu, X-M., Zhang, D-R.: Decisionmaking and Prepotent Response Inhibition Functions in Excessive Internet Users. CNS Spectrums, 14(2), 75-81 (2009)

16. Kirby, B., Dapore, A., Ash, K., Malley, K., \& West, R. (2020). Smartphone Pathology, Agency, and Reward Processing. In: Davis, F.D. et al. (eds.) Information Systems and Neuroscience, Lecture Notes in Information Systems and Organisation, 31, 321-329 (2020)

17. Horvath, J., Mundinger, C., Schmitgen, M. M., Wolf, N. D., Sambataro, F., Hirjak, D., et al.: Structural and Functional Correlates of Smartphone Addiction. Addict. Behav. 105, 106334 (2020)

18. Bechara, A.: Decision Making, Impulse Control and Loss of Willpower to Resist Drugs: A Neurocognitive Perspective. Nat. Neurosci. 8, 1458-1463 (2005)

19. Baumeister, R., Vohs, K., Tice, D.: The strength Model of Self-control. Curr. Dir. Psychol. Sci. 16, 351-355 (2007)

20. Hu, Q., West, R., Smarandescu, L.: The Role of Self-control in Information Security Violations: Insights From a Cognitive Neuroscience Perspective. J. Manag. Inf. Syst. 31, 6-48 (2015)

21. Tangney, J.P., Baumeister, R.F., Boone, A.L.: High Self-control Predicts Good Adjustment, Less Pathology, Better Grades, and Interpersonal Success. J. Pers. 72, 271-322 (2004)

22. Rueda, M., Posner, M., Rothbart, M.: Attentional Control and Self-regulation. In: Baumeister, R., Vohs, K. (eds.) Handbook of Self-Regulation: Research, Theory, and Applications, pp. 283-300. The Guilford Press, New York (2004)

23. Király, O., Griffiths, M., Urbán, R., Farkas, J., Kökönyei, G., Elekes, Z., Tamás, D., Demetrovics, Z.: Problematic Internet Use and Problematic Online Gaming Are Not the Same: Findings from a Large Nationally Representative Adolescent Sample. Cyberpsychol Behav Soc Netw, 17, 749-754 (2014)

24. Pawlikowski, M., Alstötter-Gleich, C., Brand, M.: Validation and Psychometric Properties of a Short Version of Young's Internet Addiction Test. Comput. Hum. Behav. 29, 1212-1223 (2013)

25. Kwon, Min., Kim, D., Cho, H., Yang, S.: The Smartphone Addiction Scale: Development and Validation of a Short Version for Adolescents. PLOS ONE 8(12) e83558 (2013)

26. Andreassen, C. S., Billieux, J., Griffiths, M. D., Kuss, D. J., Demetrovics, Z., Mazzoni, E., Pallesen, S.:. The Relationship Between Addictive Use of Social Media and Video Games and Symptoms of Psychiatric Disorder: A Large-scale Cross-sectional Study. Psychol. Addict. Behav. 30(2), 252-262 (2016)

27. Henry, J., Crawford, J.: The Short-form Version of the Depression Anxiety Stress Scales (DASS-21): Construct Validity and Normative data in a Large Nonclinical Sample. Brit. J. Clin. Psychol. 44, 227-239 (2005)

28. Broadbent, D., Cooper, P., FitzGerald, P., Parkes, K.: The Cognitive Failures Questionnaire (CFQ) and Its Correlates. Brit. J. Clin. Psychol. 21, 1-16 (1982)

29. Schwarzer, R., Jerusalem, M.: Generalized Self-efficacy Scale. In J Weinman, S Wright, \& M Johnston. Measures in health psychology: A user's portfolio. Causal and control beliefs. Windsor, England: NFER-NELSON, 35-37 (1995) 
30. Duckworth, A., Quinn, P.: Development and Validation of the Short Grit Scale (Grit-S). J. Pers. Assess. 91, 166-174 (2009)

31. Svante, W., Esbensen, K., Galadi, P.: Principal Component Analysis: Chemometrics and intelligent laboratory systems, 2(1-3), 37-52 (1987)

32. West, R., Budde, E., Hu, Q.: Neural Correlates of Decision Making Related to Information Security: Self-control and Moral Potency. PLoS ONE 14(9): e0221808 (2019)

33. Yves, R.: lavaan: An R Package for Structural Equation Modeling. J. Stat. Softw. 48(2), 1-36 (2012)

34. Preacher, K. J., Hayes, A. F.: Asymptotic and Resampling Strategies for Assessing and Comparing Indirect Effects in Multiple Mediator Models. Behav. Res. Methods 40, 879-891 (2008)

35. Maier, C.: Overcoming pathological IT use: How and why IT addicts terminate their use of games and social media. Int. J. Inf. Manage. 51, 1-9, 102053 (2020)

36. Alrobia, A., Mcalaney, J., Dogan, H., Phalp, K., Ali, R.: Exploring the Requirements and Design of Persuasive Intervention Technology to Combat Addiction. $6^{\text {th }}$ International Conference on Human-Centered Software Engineering (HCSE) $/ 8^{\text {th }}$ International Conference on Human Error, Safety, and System Development (HESSD), Aug 2016, Stockholm, Sweden. 130-150 (2016) 